\title{
An Arbitrary (Fractional) Orders Differential Equation with Internal Nonlocal and Integral Conditions
}

\author{
Ahmed El-Sayed ${ }^{1}$, E. O. Bin-Taher ${ }^{2}$ \\ ${ }^{1}$ Faculty of Science, Alexandria University, Alexandria, Egypt \\ ${ }^{2}$ Faculty of Science, Hadhramout University of Science and Technology, Hadhramout, Yemen \\ E-mail: amasayed@hotmail.com, ebtsamsam@yahoo.com \\ Received February 28, 2011; revised March 25, 2011; accepted March 30, 2011
}

Abstract

In this paper we study the existence of solution for the differential equation of arbitrary ( fractional) orders $\frac{\mathrm{d} x}{\mathrm{~d} t}=f\left(t, D^{\alpha} x\right), t \in(0,1)$, with the general form of internal nonlocal condition $\sum_{k=1}^{m} a_{k} x\left(\tau_{k}\right)=\beta \sum_{j=1}^{p} b_{j} x\left(\eta_{j}\right)$, $\tau_{k} \in(a, c) \subseteq(0,1), \eta_{j} \in(d, b) \subseteq(0,1), c \leq d$. The problem with nonlocal integral condition will be studied.

Keywords: Internal Nonlocal Problem, Integral Condition, Fractional Calculus, Existence of Solution, Caratheodory Theorem

\section{Introduction}

Problems with non-local conditions have been extensively studied by several authors in the last two decades. The reader is referred to ([1-10]), and references therein.

In this work we study the existence of at least one solution for the nonlocal problem of the arbitrary (fractional) order differential equation

$$
\frac{\mathrm{d} x(t)}{\mathrm{d} t}=f\left(t, D^{\alpha} x(t)\right), \quad t \in(0,1) \text { and } \alpha \in(0,1]
$$

with the general nonlocal condition

$$
\sum_{k=1}^{m} a_{k} x\left(\tau_{k}\right)=\beta \sum_{j=1}^{p} b_{j} x\left(\eta_{j}\right),
$$

where $\quad \tau_{k} \in(a, c) \subseteq(0,1), \eta_{j} \in(d, b) \subseteq(0,1), c \leq d \quad$ and $\beta \geq 0$ is parameter.

As an application, we deduce the existence of solution for the nonlocal problem of the differential (1) with the integral condition

$$
\int_{a}^{c} x(s) \mathrm{d} s=\beta \int_{d}^{b} x(s) \mathrm{d} s .
$$

It must be noticed that the following nonlocal and integral conditions are special cases of our nonlocal and integral conditions

$$
x(\tau)=\beta x(\eta), \tau \in(a, c) \text { and } \eta \in(d, b),
$$

$$
\begin{gathered}
\sum_{k=1}^{m} a_{k} x\left(\tau_{k}\right)=\beta x(\eta), \tau_{k} \in(a, c) \text { and } \eta \in(d, b), \\
\sum_{k=1}^{m} a_{k} x\left(\tau_{k}\right)=0, \tau_{k} \in(a, c), \\
\int_{a}^{c} x(s) \mathrm{d} s=\beta x(\eta), \eta \in(d, b),
\end{gathered}
$$

and

$$
\int_{a}^{c} x(s) \mathrm{d} s=0,(a, c) .
$$

\section{Preliminaries}

Let $L^{1}(I)$ denotes the class of Lebesgue integrable functions on the interval $I=[a, b]$, with the norm $\|u\|_{L^{1}}=\int_{I}|u(t)| \mathrm{d} t$ and $C(I)$ denotes the class of continuous functions on the interval $I$, with the norm $\|u\|=\sup _{t \in I}|u(t)|$ and $\Gamma($.$) denotes the gamma func-$ tion.

Definition 2.1 The fractional-order integral of the function $f \in L^{1}[a, b]$ of order $\beta \in R^{+}$is defined by (see [11])

$$
I_{a}^{\beta} f(t)=\int_{a}^{t} \frac{(t-s)^{\beta-1}}{\Gamma(\beta)} f(s) \mathrm{d} s .
$$

Definition 2.2 The Caputo fractional-order derivative of 
order $\alpha \in(0,1]$ of the absolutely continuous function $f(t)$ is defined by (see [11] and [12])

$$
D_{a}^{\alpha} f(t)=I_{a}^{1-\alpha} \frac{\mathrm{d}}{\mathrm{d} t} f(t) .
$$

Definition 2.3 The function $f:[0,1] \times R \rightarrow R$ is called $L^{1}$ - Caratheodory if

1) $t \rightarrow f(t, x)$ is measurable for each $x \in R$,

2) $x \rightarrow f(t, x)$ is continuous for almost all $t \in[0,1]$,

3) there exists $m \in L^{1}([0,1], D), D \subset R$ such that $|f| \leq m$.

Now we state Caratheodory Theorem ([13]).

Theorem 2.1 Let $f[0,1] \times R \rightarrow R$ be $L^{1}$ - Caratheodory, then the initial-value problem

$$
\frac{\mathrm{d} x(t)}{\mathrm{d} t}=f(t, x(t)) \text {, for a.e. } \mathrm{t}>0 \text {, and } x(0)=x_{o}
$$

has at least one absolutely continuous solution $x \in A C[0, T]$.

Here we generalize Caratheodory theorem for the nonlocal problem (1) - (2).

\section{Main Results}

Consider firstly the fractional-order integral equation

$$
y(t)=I^{1-\alpha} f(t, y(t)),
$$

Definition 3.1 The function $y$ is called a solution of the fractional-order integral Equation (10), if $y \in C[0,1]$ and satisfies (10).

Theorem 3.1 Let $f:[0,1] \times R \rightarrow R$ be $L^{1}$ - Cara theodory. Then there exists at least one solution of the fractional-order integral Equation (10).

Proof. Let

$M=\operatorname{Max}\left\{I_{a}^{\beta} m(t): t \in(0,1), a \geq 0\right.$ and $\left.\beta \in(0,1)\right\}$, then

$$
\begin{gathered}
\left|I_{a}^{\beta} f(t, y(t))\right| \leq \int_{a}^{t} \frac{(t-s)^{\beta-1}}{\Gamma(\beta)}|f(s, y(s))| \mathrm{d} s \\
\leq \int_{a}^{t} \frac{(t-s)^{\beta-1}}{\Gamma(\beta)} m(s) \mathrm{d} s \leq M, a \geq 0 .
\end{gathered}
$$

Define the sequence $\left\{y_{n}(t)\right\}$ by

$$
y_{n+1}(t)=\int_{0}^{t} \frac{(t-s)^{-\alpha}}{\Gamma(1-\alpha)} f\left(s, y_{n}(s)\right) \mathrm{d} s, t \in[0,1]
$$

which can be written in the operator form

$$
y_{n+1}(t)=I^{1-\alpha-\beta} I^{\beta} f\left((t), y_{n}(t)\right) .
$$

Then

$$
\begin{aligned}
& \left|y_{n+1}(t)\right| \leq I^{1-\alpha-\beta}\left|I^{\beta} f\left(t, y_{n}(t)\right)\right| \leq M \int_{0}^{t} \frac{(t-s)^{-\alpha-\beta}}{\Gamma(1-\alpha-\beta)} \mathrm{d} s \\
& \leq M \frac{(t)^{1-\alpha-\beta}}{\Gamma(2-\alpha-\beta)} \leq \frac{M}{\Gamma(2-\alpha-\beta)}
\end{aligned}
$$

For $t_{1}, t_{2} \in[0,1]$ such that $t_{1}<t_{2}$, then

$$
\begin{aligned}
& y_{n+1}\left(t_{2}\right)-y_{n+1}\left(t_{1}\right)=\int_{0}^{t_{2}} \frac{\left(t_{2}-s\right)^{-\alpha}}{\Gamma(1-\alpha)} f\left(s, y_{n}(s)\right) \mathrm{d} s \\
& -\int_{0}^{t_{1}} \frac{\left(t_{1}-s\right)^{-\alpha}}{\Gamma(1-\alpha)} f\left(s, y_{n}(s)\right) \mathrm{d} s \\
& =\int_{0}^{t_{1}} \frac{\left(t_{2}-s\right)^{-\alpha}}{\Gamma(1-\alpha)} f\left(s, y_{n}(s)\right) \mathrm{d} s \\
& +\int_{t_{1}}^{t_{2}} \frac{\left(t_{2}-s\right)^{-\alpha}}{\Gamma(1-\alpha)} f\left(s, y_{n}(s)\right) \mathrm{d} s \\
& -\int_{0}^{t_{1}} \frac{\left(t_{1}-s\right)^{-\alpha}}{\Gamma(1-\alpha)} f\left(s, y_{n}(s)\right) \mathrm{d} s \\
& \leq \int_{0}^{t_{1}} \frac{\left(t_{1}-s\right)^{-\alpha}}{\Gamma(1-\alpha)} f\left(s, y_{n}(s)\right) \mathrm{d} s \\
& +\int_{t_{1}}^{t_{2}} \frac{\left(t_{2}-s\right)^{-\alpha}}{\Gamma(1-\alpha)} f\left(s, y_{n}(s)\right) \mathrm{d} s \\
& -\int_{0}^{t_{1}} \frac{\left(t_{1}-s\right)^{-\alpha}}{\Gamma(1-\alpha)} f\left(s, y_{n}(s)\right) \mathrm{d} s .
\end{aligned}
$$

Therefore

$$
\begin{aligned}
& \left|y_{n+1}\left(t_{2}\right)-y_{n+1}\left(t_{1}\right)\right| \leq \int_{t_{1}}^{t_{2}} \frac{\left(t_{2}-s\right)^{-\alpha}}{\Gamma(1-\alpha)} m(s) \mathrm{d} s \\
& \leq \int_{t_{1}}^{t_{2}} \frac{\left(t_{2}-\theta\right)^{-\alpha}}{\Gamma(1-\alpha)} m(\theta) \mathrm{d} \theta \leq M \int_{t_{1}}^{t_{2}} \frac{\left(t_{2}-\theta\right)^{-\alpha-\beta}}{\Gamma(1-\alpha-\beta)} \mathrm{d} \theta \\
& \leq M \frac{\left(t_{2}-t_{1}\right)^{1-\alpha-\beta}}{\Gamma(2-\alpha-\beta)} .
\end{aligned}
$$

Hence $\left|t_{2}-t_{1}\right|<\delta \Rightarrow\left|y_{n+1}\left(t_{2}\right)-y_{n+1}\left(t_{1}\right)\right|<\varepsilon(\delta)$ and $\left\{y_{n}(t)\right\}$ is a sequence of equi-continuous and uniformly bounded functions. By Arzela-Ascoli Theorem, ([14] and [15]) there exists a subsequence $\left\{y_{n_{k}}(t)\right\}$ of continuous functions which converges uniformly to a continuous function $y$ as $k \rightarrow \infty$.

Now we show that this limit function is the required solution.

Since

$$
\left|f\left(s, y_{n_{k}}(s)\right)\right| \leq m(s) \in L^{1},
$$

and $f\left(s, y_{n_{k}}(s)\right)$ is continuous in the second argument, 
i.e. $f\left(s, y_{n_{k}}(s)\right) \rightarrow f(s, y(s))$ as $k \rightarrow \infty$,

therefore the sequence $\left\{(t-s)^{-\alpha} f\left(s, y_{n_{k}}(s)\right)\right\}$, $\alpha \in(0,1)$ satisfies Lebesgue dominated convergence theorem. Hence

$$
\begin{aligned}
& \lim _{k \rightarrow \infty} \int_{0}^{t} \frac{(t-s)^{-\alpha}}{\Gamma(1-\alpha)} f\left(s, y_{n_{k}}(s)\right) \mathrm{d} s \\
& =\int_{0}^{t} \frac{(t-s)^{-\alpha}}{\Gamma(1-\alpha)} f(s, y(s)) \mathrm{d} s=y(t),
\end{aligned}
$$

which proves the existence of at least one solution $y \in C[0,1]$ of the fractional-order functional integral Equation (10).

For the existence of solution for the nonlocal problem (1) - (2) we have the following theorem.

Theorem 3.2 Let the assumptions of Theorem 3.1 are satisfied. Then nonlocal problem (1) - (2) has at least one solution $x \in A C[0,1]$.

Proof. Consider the nonlocal problem (1) - (2).

Let $y(t)=D^{\alpha} x(t)$, then

$$
\begin{aligned}
& y(t)=I^{1-\alpha} \frac{\mathrm{d} x(t)}{\mathrm{d} t}, \\
& y(t)=I^{1-\alpha} f(t, y(t))
\end{aligned}
$$

and $y$ is the solution of the fractional-order integral Equation (10).

Operating by $I^{\alpha}$ on both sides of Equation(11), we obtain

$$
\begin{gathered}
I^{\alpha} y(t)=I \frac{\mathrm{d} x(t)}{\mathrm{d} t}=x(t)-x(0) \Rightarrow \\
x(t)=x(0)+I^{\alpha} y(t) .
\end{gathered}
$$

Let $t=\tau_{k}$ in Equation (13), we get

$$
\sum_{k=1}^{m} a_{k} x\left(\tau_{k}\right)=\sum_{k=1}^{m} a_{k} \int_{0}^{\tau_{k}} \frac{\left(\tau_{k}-s\right)^{\alpha-1}}{\Gamma(\alpha)} y(s) \mathrm{d} s+x(0) \sum_{k=1}^{m} a_{k} .
$$

And let $t=\eta_{j}$ in Equation (13), we get

$$
\sum_{j=1}^{p} b_{j} x\left(\eta_{j}\right)=\sum_{j=1}^{p} b j \int_{0}^{\eta_{j}} \frac{\left(\eta_{j}-s\right)^{\alpha-1}}{\Gamma(\alpha)} y(s) \mathrm{d} s+x(0) \sum_{j=1}^{p} b_{j} .
$$

From Equation (2), we get

$$
\begin{aligned}
& \sum_{k=1}^{m} a_{k} \int_{0}^{\tau_{k}} \frac{\left(\tau_{k}-s\right)^{\alpha-1}}{\Gamma(\alpha)} y(s) \mathrm{d} s+x(0) \sum_{k=1}^{m} a_{k} \\
& =\beta \sum_{j=1}^{p} b_{j} \int_{0}^{\eta_{j}} \frac{\left(\eta_{j}-s\right)^{\alpha-1}}{\Gamma(\alpha)} y(s) \mathrm{d} s+x(0) \beta \sum_{j=1}^{p} b_{j} .
\end{aligned}
$$

Then we get

$$
\begin{aligned}
& x(0)=A\left(\sum_{k=1}^{m} a_{k} \int_{0}^{\tau_{k}} \frac{\left(\tau_{k}-s\right)^{\alpha-1}}{\Gamma(\alpha)} y(s) \mathrm{d} s\right. \\
&\left.-\beta \sum_{j=1}^{p} b_{j} \int_{0}^{\eta_{j}} \frac{\left(\eta_{j}-s\right)^{\alpha-1}}{\Gamma(\alpha)} y(s) \mathrm{d} s\right)
\end{aligned}
$$

and

$$
\begin{aligned}
x(t)= & A\left(\sum_{k=1}^{m} a_{k} \int_{0}^{\tau_{k}} \frac{\left(\tau_{k}-s\right)^{\alpha-1}}{\Gamma(\alpha)} y(s) \mathrm{d} s\right. \\
& \left.\quad-\beta \sum_{j=1}^{p} b_{j} \int_{0}^{\eta_{j}} \frac{\left(\eta_{j}-s\right)^{\alpha-1}}{\Gamma(\alpha)} y(s) \mathrm{d} s\right) \\
& +\int_{0}^{t} \frac{(t-s)^{\alpha-1}}{\Gamma(\alpha)} y(s) \mathrm{d} s
\end{aligned}
$$

where

$$
A=\left(\beta \sum_{j=1}^{p} b_{j}-\sum_{k=1}^{m} a_{k}\right)^{-1}
$$

which, by Theorem 3.1, has at least one solution $x \in A C(0,1)$.

Now, from Equation (15), we have

$$
\begin{aligned}
& x(0)=\lim _{t \rightarrow 0^{+}} x(t)=A \sum_{k=1}^{m} a_{k} \int_{0}^{\tau_{k}} \frac{\left(\tau_{k}-s\right)^{\alpha-1}}{\Gamma(\alpha)} y(s) \mathrm{d} s \\
& -A \beta \sum_{j=1}^{p} b_{j} \int_{0}^{\eta_{j}} \frac{\left(\eta_{j}-s\right)^{\alpha-1}}{\Gamma(\alpha)} y(s) \mathrm{d} s
\end{aligned}
$$

and

$$
\begin{aligned}
& x(1)=\lim _{t \rightarrow 1^{-}} x(t)=A \sum_{k=1}^{m} a_{k} \int_{0}^{\tau_{k}} \frac{\left(\tau_{k}-s\right)^{\alpha-1}}{\Gamma(\alpha)} y(s) \mathrm{d} s \\
& -A \beta \sum_{j=1}^{p} b_{j} \int_{0}^{\eta_{j}} \frac{\left(\eta_{j}-s\right)^{\alpha-1}}{\Gamma(\alpha)} y(s) \mathrm{d} s+\int_{0}^{1} \frac{(1-s)^{\alpha-1}}{\Gamma(\alpha)} y(s) \mathrm{d} s
\end{aligned}
$$

from which we deduce that Equation (15) has at least one solution $x \in A C[0,1]$.

To complete the proof, differentiating (15), we obtain

$$
\frac{\mathrm{d} x}{\mathrm{~d} t}=y(t)=f\left(t, D^{\alpha} x(t)\right) .
$$

Also from (15) we can prove that the solution satisfies the nonlocal condition (2).

\section{Nonlocal Integral Condition}

Let $x \in A C[0,1]$. be the solution of the nonlocal problem (1) - (2).

Let $a_{k}=t_{k}-t_{k-1}, \tau_{k} \in\left(t_{k-1}, t_{k}\right), a=t_{0}<t_{1}<t_{2}, \cdots<t_{m}=c$ 
and $b_{j}=s_{j}-s_{j-1}, \eta_{j} \in\left(s_{j-1}, s_{j}\right), d=s_{0}<s_{1}<s_{2}, \cdots<s_{p}=b$ then the nonlocal condition (2) will be

$$
\sum_{k=1}^{m}\left(t_{k}-t_{k-1}\right) \times\left(\tau_{k}\right)=\beta \sum_{j=1}^{p}\left(s_{j}-s_{j-1}\right) \times\left(\eta_{j}\right) .
$$

From the continuity of the solution $x$ of the nonlocal problem (1) - (2) we can obtain

$$
\lim _{m \rightarrow \infty} \sum_{k=1}^{m}\left(t_{k}-t_{k-1}\right) x\left(\tau_{k}\right)=\beta \lim _{p \rightarrow \infty} \sum_{j=1}^{p}\left(s_{j}-s_{j-1}\right) \times\left(\eta_{j}\right) .
$$

and the nonlocal condition (2) transformed to the integral one

$$
\int_{a}^{c} x(s) \mathrm{d} s=\beta \int_{d}^{b} x(s) \mathrm{d} s .
$$

Now, we have the following Theorem

Theorem 4.1 Let the assumptions of Theorem 3.2 are satisfied. Then there exist at least one solution $x \in A C[0,1]$. of the nonlocal problem with integral condition,

$$
\begin{gathered}
x^{\prime}(t)=f\left(t, D^{\alpha} x(t)\right), t \in(0,1), \\
\int_{a}^{c} x(s) \mathrm{d} s=\beta \int_{d}^{b} y(s) \mathrm{d} s, \beta(b-d) \neq(c-a) .
\end{gathered}
$$

Letting $\beta=0$ in (16), the we can easily prove the following corollary .

Theorem 4.2 Let the assumptions 1) - 2) are satisfied. Then the nonlocal problem

$$
\begin{gathered}
x^{\prime}(t)=f\left(t, D^{\alpha} x(t)\right), t \in(0,1), \\
\int_{a}^{c} x(s) \mathrm{d} s=0, \quad(a, c) \subset(0,1)
\end{gathered}
$$

has at least one solution $x \in A C[0,1]$.

\section{References}

[1] A. Boucherif, "First-Order Differential Inclusions with Nonlocal Initial Conditions," Applied Mathematics Letters, Vol. 15, No. 4, 2002, pp. 409-414. doi:10.1016/S0893-9659(01)00151-3

[2] A. Boucherif, "Nonlocal Cauchy Problems for First-Order Multivalued Differential Equations," Electronic Journal of Differential Equations, Vol. 2002, No. 47, 2002, pp. 1-9.

[3] A. Boucherif and R. Precup, "On the Nonlocal Initial
Value Problem for First Order Differential Equations,” Fixed Point Theory, Vol. 4, No. 2, 2003, pp. 205-212.

[4] A. Boucherif, "Semilinear Evolution Inclusions with Nonlocal Conditions," Applied Mathematics Letters, Vol. 22, No. 8, 2009, pp. 1145-1149. doi:10.1016/j.aml.2008.10.004

[5] M. Benchohra, E. P. Gatsori and S. K. Ntouyas, "Existence Results for Seme-Linear Integrodifferential Inclusions with Nonlocal Conditions," Rocky Mountain Journal of Mathematics, Vol. 34, No. 3, Fall 2004.

[6] M. Benchohra, S. Hamani and S. K. Ntouyas, "Boundary Value Problems for Differential Equations with Fractional Order and Nonlocal Conditions," Nonlinear Analysis: Theory, Methods \& Applications, Vol. 71, No. 7-8, 2009, pp. 2391-2396. doi:10.1016/j.na.2009.01.073

[7] A. M. A. El-Sayed and Sh. A. Abd El-Salam, "On the Stability of a Fractional Order Differential Equation with Nonlocal Initial Condition,” Electronic Journal of Qualitative Theory of Differential Equations, Vol. 2008, No. 29, 2008, pp. 1-8.

[8] A. M. A. El-Sayed and E. O. Bin-Taher, "A Nonlocal Problem of an Arbitrary (Fractional) Orders Differential Equation,” Alexandria Journal of Mathematics, Vol. 1, No. 2, 2010, pp. 1-7.

[9] E. Gatsori, S. K. Ntouyas and Y. G. Sficas, "On a Nonlocal Cauchy Problem for Differential Inclusions," Abstract and Applied Analysis, Vol. 2004, No. 5, 2004, pp. 425-434.

[10] G. M. N’Guérékata, “A Cauchy Problem for Some Fractional Abstract Differential Equation with Non Local Conditions," Nonlinear Analysis: Theory, Methods \& Applications, Vol. 70, No. 5, 2009, pp. 1873-1876. doi:10.1016/j.na.2008.02.087

[11] I. Podlubny, "Fractional Differential Equations," Academic Press, San Diego, New York and London, 1999.

[12] I. Podlubny and A. M. A. EL-Sayed, "On Two Definitions of Fractional Calculus,” Preprint UEF 03-96, ISBN 80-7099-252-2, Institute of Experimental Physics, Slovak Academy of Science, 1996.

[13] R. F. Curtain and A. J. Pritchard, "Functional Analysis in Modern Applied Mathematics,” Academic Press, London, 1977.

[14] K. Deimling, “Nonlinear Functional Analysis,” SpringerVerlag, Berlin, 1985.

[15] J. Dugundji and A. Granas, "Fixed Point Theory," Monografie Mathematyczne, Polska Akademia Nauk, Warszawa, Vol. 1, 1982. 